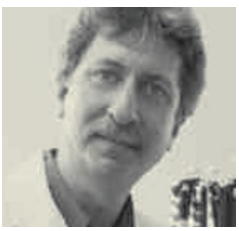

Bruce L. Levine

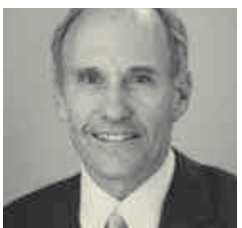

Carl H. June

\title{
Assembly line immunotherapy
} Bruce L. Levine and Carl H. June explore how to make engineered immune cells that can eradicate cancer widely available.

$\mathrm{T}$ The myth of the chimaera, a beast that is part lion, part goat and part serpent, originated in ancient Lycia. Two millennia later and a few hundred kilometres to the south, scientists at the Weizmann Institute of Science in Rehovot, Israel, described a chimaeric receptor expressed in immune cells called T cells. This chimaera - part antibody and part T-cell receptor - can be designed to recognize and destroy any tumour.

T cells with these chimaeric antigen receptors (CARs) can eradicate kilograms of leukaemia in a few weeks in people whose cancers didn't succumb to the best available conventional therapies. But so far, this experimental therapy has been available only to small numbers of people in pilot trials at academic centres.

The central question now is whether this technique be scaled up to treat thousands of patients.

We think that by making use of equipment and facilities developed for blood banks and stem-cell laboratories, and by automating production, it will be possible to make these T-cell therapies widely available to the many people who need it. Indeed, thanks to this innovation, leukaemia treatment could be on the brink of an evolution similar to the one the automotive industry experienced in recent decades in its transition from manual assembly lines to robotic automation ${ }^{1}$.

When the technique was first devised, it required manual selection of tumourspecific $\mathrm{T}$ cells before their numbers were increased in the lab by repeatedly stimulating them with specific tumour antigens. But this process requires expensive materials and takes weeks or months to generate billions of $\mathrm{T}$ cells.

In 1996, we reported an advance in culture technology that can efficiently produce large populations of T cells, approximating the mass of $\mathrm{T}$ cells in the human immune system ${ }^{2}$. We attached two antibodies to cell-sized magnetic beads, and used these antibodies to deliver two signals needed for T-cell expansion. This process enabled us to generate sufficient quantities of T cells within ten days.

We and others have used this method to treat several hundred people enrolled in clinical trials for leukaemias and other cancers. In the past few years, this approach, combined with CARs, has produced remission in more than half the adults and children treated who have advanced acute and chronic lymphoid leukaemia ${ }^{3,4}$

Many scientists have raised legitimate concerns about the perceived complexity of this type of therapy and its broad applicability. Some have dismissed it as a fringe or boutique therapy that would be

ONATURE.COM For some of the latest research on T-cell therapies: go.nature.com/zk2img impossible to commercialize ${ }^{5}$. Also, regulations set up by the US Food and Drug Administration and the European Medicines Agency are largely intended for drugs prepared and tested far in advance of medical need, and for which a single production run serves thousands of patients. Furthermore, the pharmaceutical industry's own quality control systems are geared towards generating large batches of drugs of consistent quality.

So, how can manufacturing be optimized, and how should consistency be judged when producing truly individualized medical therapies? The answer, we believe, lies in the creative use of existing facilities and a concerted effort to build the required infrastructure.

As a historical example, organ transplants, and bone-marrow and stem-cell transplants, were seen as exotic a few decades ago. Today, these therapies are accessible to tens of thousands of people. Conventional drugs are scaled up to large batches, but these personalized therapies were 'scaled out' to multiple medical centres with technically advanced operating theatres, laboratories and medical staff.

Equipment developed for blood banks and stem-cell laboratories is now routinely used in advanced T-cell therapy laboratories. In the short term, T-cell therapies will be produced in specialized cell-therapy production facilities located throughout the world at academic medical centres and biotechnology companies. Still, the current cell culture process requires extensive manipulation by highly skilled scientists and technicians. How will $\mathrm{T}$-cell therapies achieve the mass production needed to treat large numbers of patients?

As CAR T-cell processing also becomes more automated, we will be able to efficiently produce individualized therapies for larger numbers of people. But technology alone cannot solve this problem. Regulations and standards must evolve to reflect products that are personalized and sometimes administered in settings of urgent medical need.

Several pharmaceutical and biotechnology companies have entered this field in recent months with the aim of developing commercialscale manufacturing of CAR T cells for worldwide application. We are optimistic that the resources and expertise of the pharmaceutical industry will create the infrastructure needed for the widespread availability of this disruptive technology. Developing engineered T-cell therapies in large numbers will be challenging, but it is justified given their power to treat cancer.

Bruce L. Levine is an associate professor in cancer gene therapy and Carl H. June is a professor of immunotherapy at the University of Pennsylvania's Perelman School of Medicine in Philadelphia.

\footnotetext{
1. Michalos, G. et al. CIRP J. Manuf. Sci Technol. 2, 81-91 (2010)

2. Levine, B. L. et al. Science 272, 1939-1943 (1996).

3. Grupp, S. A. et al. N. Engl. J. Med. 368, 1509-1518 (2013).

4. Porter, D. L. et al. N. Engl. J. Med. 365, 725-733 (2011).

5. Baker, M. Nature Med. 17, 519 (2011).

The authors declare a conflict of interest: go.nature/rpnw28
} 